\title{
Strömungsfeldmessung der Kühlschmierstoffzufuhr an der Schleifscheibe
}

\author{
Flow field measurement of the cooling lubricant supply at the grinding wheel
}

https://doi.org/10.1515/teme-2021-0098

Eingang 26. August 2021; angenommen 19. Oktober 2021

Zusammenfassung: Kühlschmierstoff (KSS)-strömungen werden in Schleifprozessen eingesetzt, um Schleifbrand am Werkstück zu verhindern. Bislang wurde die KSSZufuhr heuristisch optimiert. Um eine zielgerichtete KSSStrömungsoptimierung $\mathrm{zu}$ ermöglichen, wird erstmals die optische schleifscheibennahe Messung von KSSStrömungsfeldern mittels Particle Image Velocimetry (PIV) und durch Nachverfolgung von Mustern in Schattenwurfbildern untersucht. Als erster Schritt wird an der rotierenden Schleifscheibe, jedoch ohne die Bearbeitung eines Werkstücks, gemessen. Beide Messmethoden liefern vergleichbare Ergebnisse, wobei nahe der Schleifscheibenoberfläche mittels PIV Messwerte mit geringerer Messunsicherheit erzielt werden. Mit dem verwendeten Messaufbau lassen sich mittels PIV Geschwindigkeiten in einem Abstand zwischen $0 \mathrm{~cm}$ bis $2 \mathrm{~cm}$ und mittels Schattenwurf zwischen $0 \mathrm{~cm}$ bis $5 \mathrm{~cm}$ zur Schleifscheibenoberfläche messen. Im Ergebnis konnte erstmals der für die Kühlleistung relevante Parameter des Geschwindigkeitsverhältnisses zwischen KSS-Anströmung und Schleifscheibenoberflächengeschwindigkeit in situ gemessen

\footnotetext{
*Korrespondenzautor: Christoph Vanselow, Universität Bremen, Bremer Institut für Messtechnik, Automatisierung und Qualitätswissenschaft (BIMAQ), Linzer Str. 13, 28359 Bremen, Germany, E-Mail: c.vanselow@bimaq.de, ORCID: https://orcid.org/0000-0002-1233-4476

Björn Espenhahn, Universität Bremen, Bremer Institut für Messtechnik, Automatisierung und Qualitätswissenschaft (BIMAQ), Linzer Str. 13, 28359 Bremen, Germany

Lukas Schumski, Leibniz-Institut für Werkstofforientierte Technologien (IWT), Badgasteiner Straße 3, 28359 Bremen, Germany Dirk Stöbener, Andreas Fischer, Universität Bremen, Bremer Institut für Messtechnik, Automatisierung und Qualitätswissenschaft (BIMAQ), Linzer Str. 13, 28359 Bremen, Germany; und Universität Bremen, MAPEX Center for Materials and Processe, Postbox 330440 , 28359 Bremen, Germany, ORCID:

https://orcid.org/0000-0002-1624-2106 (D. Stöbener), https://orcid.org/0000-0001-7349-7722 (A. Fischer)

Daniel Meyer, Leibniz-Institut für Werkstofforientierte Technologien (IWT), Badgasteiner Straße 3, 28359 Bremen, Germany; und Universität Bremen, MAPEX Center for Materials and Processe, Postbox 330440, 28359 Bremen, Germany
}

werden. Es wurde festgestellt, dass eine aus der Literatur bekannte Verringerung der Kühleffizienz ab einem Geschwindigkeitsverhältnis von 0,8 mit einem Abprallen des KSS-Strahls beim Auftreffen auf die Schleifscheibe korreliert. In zukünftigen Untersuchungen ist zu klären, wie sich die hier gewonnenen Erkenntnisse zur Messfähigkeit und zum Strömungsverhalten auf den Schleifprozess mit Werkstück übertragen lassen.

Schlagwörter: Zweiphasenströmungen, Particle Image Velocimetry, Optische Strömungsmesstechnik, Schleifenprozess.

Abstract: Cooling lubricant (coolant) flows are used in grinding processes to prevent grinding burn on the workpiece. So far, the coolant supply has been optimized heuristically. To enable a target-oriented coolant flow optimization, the optical measurement of coolant flow fields close to the grinding wheel by means of particle image velocimetry (PIV) and by tracing patterns in shadowgraphs is investigated for the first time. As a first step, measurements are made on the rotating grinding wheel, but without machining a workpiece. Both measurement methods provide comparable results, whereby measurement values with lower measurement uncertainty are obtained close to the grinding wheel surface by means of PIV. With the measurement setup used, velocities can be measured at a distance between $0 \mathrm{~cm}$ to $2 \mathrm{~cm}$ from the grinding wheel surface using PIV and between $0 \mathrm{~cm}$ to $5 \mathrm{~cm}$ using shadowgraphs. As a result, the parameter of the velocity ratio between the coolant incident jet and the grinding wheel surface velocity, which is relevant to the cooling performance, could be measured in situ for the first time. It was found that a reduction in cooling efficiency known from the literature above a velocity ratio of 0.8 correlates with a bouncing of the coolant jet when it hits the grinding wheel. In future investigations, it must be clarified how the knowledge gained here on the measurement capability and flow behavior can be transferred to the grinding process with workpiece.

Keywords: Two phase flows, Particle Image Velocimetry, optical flow measurement, grinding. 


\section{Einleitung}

Schleifprozesse sind ein wichtiger Fertigungsschritt für metallische Bauteile, wobei Kühlschmierstoff (KSS)-Strömungen zur Schmierung und Kühlung dieser Prozesse eingesetzt werden. Ziel dabei ist eine thermische Schädigung des Werkstücks aufgrund von Schleifbrand zu vermeiden und die Abnutzung der Schleifscheibe $\mathrm{zu}$ verringern. Eine Optimierung der Strömungsbedingungen des KSS in Bezug auf eine effiziente Kühlung des Werkstücks würde eine Verbesserung der Oberflächengüte und eine Schonung von Ressourcen durch eine Verlängerung der Lebensdauer des Werkzeugs und eine Verringerung des KSS-Bedarfs bewirken. $\mathrm{Zu}$ diesem Zweck wurden bislang heuristische Methoden angewendet [1, 2], deren Ergebnisse nur für den jeweils untersuchten Anwendungsfall gültig sind. Für eine zielgerichtete Untersuchung der für eine effiziente Kühlung erforderlichen Strömungscharakteristika, die zu einem tieferen Verständnis der grundsätzlichen Kühlmechanismen beiträgt, wird eine nicht-invasive Messung des KSSStrömungsgeschwindigkeitsfelds nahe der rotierenden Schleifscheibe benötigt [3].

Trotz der begrenzten optischen Zugänglichkeit in Schleifmaschinen sind optische Methoden prinzipiell für nicht-invasive Strömungsfeldmessungen geeignet [4]. Sie können in dopplerbasierte und Laufzeitverfahren untergliedert werden. Für dopplerbasierte Strömungsfeldmessungen sind für die Messung einer Geschwindigkeitskomponente ein Beleuchtungs- und ein Beobachtungspfad und für jede weitere Komponente ein weiterer Beleuchtungs- oder Beobachtungspfad erforderlich [5]. Dagegen bieten Laufzeitverfahren die Möglichkeit, mittels jeweils einem Beleuchtungs- und Beobachtungspfad bereits zwei Geschwindigkeitskomponenten zu messen. Daher eignen sich aufgrund der schwierigen optischen $\mathrm{Zu}$ gänglichkeit für Messungen in der Schleifmaschine Laufzeitverfahren besonders.

Für KSS-Strömungsfeldmessungen in einer Schleifmaschine sind insbesondere Messungen nahe der Schleifscheibenoberfläche erforderlich. Die Particle Image Velocimetry (PIV) ist ein optisches Laufzeitverfahren, bei dem typischerweise in die Strömung eingebrachte Partikel als Tracer für Geschwindigkeitsfeldmessungen verfolgt werden [6]. Die Partikel werden dafür in einem Lichtschnitt mit einer typischen Dicke von etwa $1 \mathrm{~mm}$ mittels Pulslaser beleuchtet. In zwei aufeinander folgenden Partikelbildern wird der Partikelversatz in aufgeteilten Bildsegmenten mittels Kreuzkorrelation bestimmt und über den Zeitversatz der gepulsten Beleuchtung die Geschwindigkeit berechnet. In Zweiphasenströmungen wurde bei- spielsweise mittels PIV das Geschwindigkeitsfeld eines internen Dieseleinspritzvorgangs gemessen [7], wobei anstatt Partikel, die Strömungsstrukturen in Form von Tropfen als Tracer verwendet wurden. Weiterhin wurden bereits PIV-Grenzschichtmessungen an rauen, gekrümmten und bewegten Oberflächen durchgeführt $[8,9,10]$, womit schleifscheibennahe Messungen der KSS-Strömung prinzipiell möglich erscheinen. Eine Umsetzung von PIVStrömungsfeldmessungen der KSS-Zufuhr in einer Schleifmaschine erfolgte bislang jedoch noch nicht.

Äquivalent zur PIV-Auswertung wurden anstelle von Partikelbildern auch Schattenwurfbilder für Geschwindigkeitsfeldmessungen genutzt, z. B. für die Untersuchung einer Heliumdüsenströmung [11]. In den Schattenwurfbildern sind Strömungsstrukturen aufgrund von Dichtevariationen sichtbar und können als Tracer für Geschwindigkeitsmessungen genutzt werden. Die Zweiphasenströmung des zunächst zusammenhängenden KSS-Strahls zerfällt je nach verwendeter Düsengeometrie und Volumenstrom spätestens $15 \mathrm{~cm}$ nach dem Düsenaustritt [12]. Die so entstehenden Tropfen und Strömungsstrukturen sind grundsätzlich als Tracer geeignet, jedoch ist unklar, ob der entstehende Kontrast in Schattenwurfbildern eine Messung des KSS-Strömungsfeldes nahe der Schleifscheibe ermöglicht.

Zusammenfassend eignen sich PIV- und Schattenwurf Image Velocimetry (SIV)-Verfahren prinzipiell für Geschwindigkeitsfeldmessungen in einer Schleifmaschine. Da aber während eines Schleifprozesses Optiken innerhalb der Schleifmaschine nach kürzester Zeit durch KSSTropfen kontaminiert werden, ist fraglich inwiefern eine Implementierung der Strömungsmesssysteme in eine Schleifmaschine möglich ist. Insbesondere ist unklar, inwiefern die Auswertung von PIV- und SIV für Grenzschichtströmungsmessungen nahe der Schleifscheibe geeignet ist.

Daher sollen ein PIV- und ein SIV-Messsystem in eine Schleifmaschine implementiert und quantitative Strömungsfeldmessungen der KSS-Zufuhr beim Schleifen zunächst ohne Werkstück durchgeführt werden. Die gemessenen Strömungsfelder sollen verglichen werden und dabei explizit untersucht werden, inwieweit beide Techniken für schleifscheibennahe Messungen geeignet sind. Damit soll perspektivisch gezeigt werden, dass Strömungsfeldmessungen für die zielgerichtete Identifikation von kühleffizienten KSS-Strömungsphänomenen möglich sind. Dafür werden der Ansatz und die Umsetzung der PIV- und SIV-Messungen in einer Schleifmaschine in Kapitel 2 beschrieben. Die Messergebnisse werden in Kapitel 3 gezeigt und deren Abweichungen untersucht. Dabei wird außerdem quantifiziert, wie nahe Strömungsfeldmessungen an 
a)
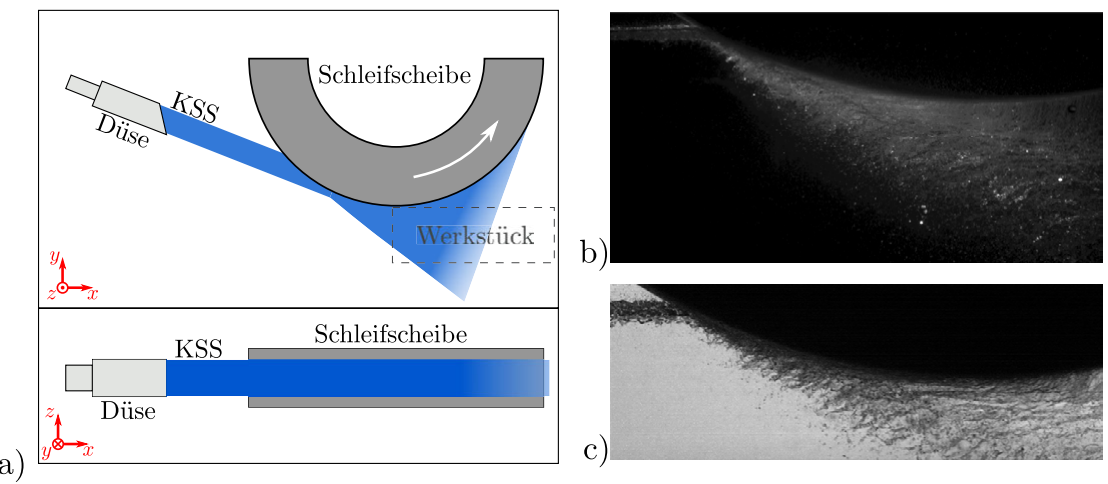

Abb. 1: a) Schematische Darstellung der KSS-Strömung an der rotierenden Schleifscheibe. b) PIV-Rohbild und c) Schattenwurf-Rohbild der KSS-Strömung an der rotierenden Schleifscheibe.

der Schleifscheibe möglich sind. Zum Schluss gibt es in Kapitel 4 eine Zusammenfassung der Ergebnisse und einen Ausblick auf zukünftige Arbeiten.

\section{Messansatz und -aufbau}

Die KSS-Strömung wird mittels PIV und über die Auswertung von Schattenwurfbildern (SIV) gemessen. Abbildung 1a zeigt dazu schematisch die KSS-Strömung an der rotierenden Schleifscheibe. Die KSS-Anströmung ist in Richtung der Umfangsgeschwindigkeit der Schleifscheibe ausgerichtet. Ziel dabei ist, dass der KSS an der Schleifscheibe anhaftet und somit mit in den Schleifprozess im Schleifspalt zwischen Schleifscheibe und Werkstück gelangt. Das Werkstück und damit der Schleifspalt befindet sich im Schleifprozess am Tiefpunkt der Schleifscheibe. Um aus messtechnischer Sicht die Machbarkeit von Strömungsfeldmessungen in der Schleifmaschine zu zeigen und zu klären inwieweit schleifscheibennahe Strömungsfeldmessungen möglich sind, wird in diesen Untersuchungen als erster Schritt prozessnah, d.h. in der Schleifmaschine bei rotierender Schleifscheibe, jedoch zunächst ohne Werkstück gemessen.

Die Messansätze PIV und SIV unterscheiden sich in der Beleuchtungsart des Fluids. So wird die PIVBeleuchtung über einen Lichtschnitt in $y$-Richtung in der $x, y$-Ebene umgesetzt, was zu einer direkten Beleuchtung des Fluids in einer Messebene führt. Als Messsignal werden Lichtstrahlen anstatt an den typischerweise verwendeten Streupartikeln an den Strömungsstrukturen in Richtung Kamera reflektiert, gebrochen oder gestreut. So werden die Strömungsstrukturen in den Kamerabildern sichtbar, siehe Abb.1b. Als komplementärer Ansatz wird bei den SIV-Messungen eine homogene Hintergrundbeleuch- tung verwendet. Durch die Störung der vom Hintergrund in Richtung Kamera laufenden Lichtstrahlen an dem Brechungsindexsprung zwischen flüssigem KSS-Fluid und der umgebenden Luft erscheinen die Strömungsstrukturen im Gegensatz zu den PIV-Aufnahmen als Schatten, siehe Abb.1c. So entsteht gewissermaßen ein inverses Bild zu den PIV-Aufnahmen, wobei jedoch im Gegensatz zur Lichtschnittbeleuchtung die gesamte Strömung in Kamerablickrichtung betrachtet wird. Die Geschwindigkeitsmessung mittels SIV erfährt somit eine räumliche Mittelung in Kamerablickrichtung. Die Geschwindigkeitsauswertung erfolgt in beiden Ansätzen analog mittels Korrelationsauswertealgorithmus. Bei der permanenten Hintergrundbeleuchtung für SIV wird eine Hochgeschwindigkeitskamera eingesetzt, wobei der zeitliche Versatz für die Geschwindigkeitsberechnung der Inversen der verwendeten Bildrate entspricht.

Die Strömungsmessungen werden in einer Schleifmaschine Micro-Cut A8 CNC von der Firma ELB durchgeführt, siehe Abb.2a. Für die Messungen wird eine kommerziell erhältliche Schleifscheibe mit einem Radius von $20 \mathrm{~cm}$ eingesetzt. Die KSS-Zufuhr ist über eine kühleffiziente Rouse-Düse [1] mit einer rechteckförmigen Öffnung von $0,9 \mathrm{~mm} \times 21 \mathrm{~mm}$ realisiert. Über die Volumenströme $Q$ von $16,6 \mathrm{~L} \mathrm{~min}^{-1}, 21,3 \mathrm{~L} \mathrm{~min}^{-1}, 26,6 \mathrm{~L} \mathrm{~min}^{-1}, 31,9 \mathrm{~L} \mathrm{~min}^{-1}$, $37,2 \mathrm{~L} \mathrm{~min}^{-1}$ und $42,5 \mathrm{~L} \mathrm{~min}^{-1}$ wird die Anströmgeschwindigkeit variiert. Bei einer konstanten Schleifscheibenumfangsgeschwindigkeit von $v_{\mathrm{SS}}=25 \mathrm{~m} \mathrm{~s}^{-1}$ wird somit das Geschwindigkeitsverhältnis $v=v_{\mathrm{KSS}} / v_{\mathrm{SS}}$ zwischen KSSStrömung und Schleifscheibe gezielt verändert, was sich als ein wesentlicher Parameter für eine Optimierung der Kühleffizienz herausgestellt hat [1].

Um einen ungestörten optischen Zugang zu erhalten, wurde die Sicherheitsscheibe der Schleifmaschine an der Tür zur Schleifkammer entfernt und eine Spritzschutzfolie 

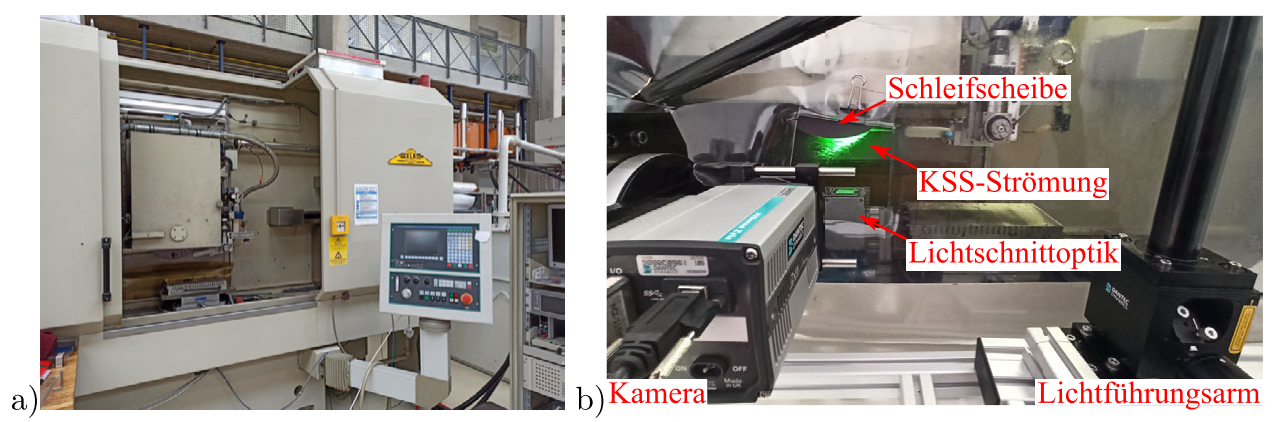

Abb. 2: a) Für die Messungen verwendete Schleifmaschine. b) Implementierung des PIV-Messsystems in der Schleifmaschine.

mit einer Aussparung für die verwendeten Kameras eingesetzt, siehe Abb.2b. Um Laser- und Arbeitsschutz zu gewährleisten, ist die Öffnung mit einem Aluminiumgehäuse gesichert. Eine sCMOS Kamera mit einer Pixelauflösung von 5,5 Mpx und einem Objektiv mit $50 \mathrm{~mm}$ Brennweite zeichnet die PIV-Bilder mit einer Messrate von $15 \mathrm{~Hz}$ auf. Dabei resultiert bei einem Arbeitsabstand von etwa $60 \mathrm{~cm}$ eine räumliche Auflösung von $80 \mu \mathrm{m}$. Die PIVLichtschnittbeleuchtung ist über einen Lichtführungsarm in die Schleifkammer eingebracht. Um Beschädigungen durch KSS-Spritzer zu vermeiden, ist der Lichtführungsarm sorgfältig abgedichtet und die am Ende liegende Lichtschnittoptik in einem dichten Gehäuse mit Fenster untergebracht. Mit einer Lichtschnittdicke von $1 \mathrm{~mm}$ wird das KSS-Fluid mit einem zeitlichen Abstand von $100 \mu$ s beleuchtet. Dafür wird ein Nd:YAG-Pulslaser mit einer Wellenlänge von $532 \mathrm{~nm}$ verwendet, dessen maximale Pulsenergie von $200 \mathrm{~mJ}$ soweit gedrosselt wird, bis es nur vereinzelt zu Überbelichtungen der Kamerabilder kommt, welche aufgrund der teilweise auftretenden direkten Reflexionen an den Strömungsstrukturen nicht komplett vermeidbar sind.

Für die SIV-Messungen wird eine Hochgeschwindigkeitskamera mit einer Pixelanzahl von $1 \mathrm{Mpx}$ und einem Objektiv mit einer Brennweite von $105 \mathrm{~mm}$ verwendet. Es resultiert bei einem Arbeitsabstand von etwa $100 \mathrm{~cm}$ eine örtliche Auflösung von $150 \mu \mathrm{m}$. Für die homogene Hintergrundbeleuchtung wird ein LED-Panel an der Rückwand der Schleifkammer installiert, welches eine Beleuchtungsstärke von $2400 \mathrm{~lm}$ liefert. Die Bildrate von $13,7 \mathrm{kHz}$ liefert einen zeitlichen Versatz zwischen den Aufnahmen von $73 \mu$ s.

\section{Messergebnisse}

In diesem Kapitel werden die Messergebnisse der PIVund SIV-Messungen gezeigt und verglichen. Zudem wer- den die Messergebnisse dahingehend untersucht, inwieweit schleifscheibennahe Messungen möglich sind. Da$\mathrm{zu}$ wird zunächst die Schichtdicke der an der Schleifscheibe anliegenden KSS-Strömung bestimmt, um zu klären, welche Anforderungen an die räumlichen Auflösung der Geschwindigkeitsfeldmessungen auftreten. Weiterhin wird die Schleifscheibenoberfläche in den PIV-Rohbildern detektiert, damit der Abstand zwischen gemessenem Geschwindigkeitsfeld und der Schleifscheibenoberfläche bestimmt werden kann.

Für eine Abschätzung der Schichtdicke der anliegenden KSS-Strömung an der Schleifscheibe und der Position der Schleifscheibenoberfläche werden die Intensitätswerte von 60 PIV-Rohbildern aufsummiert und anhand des Schwellenwerts nach Otsu [13] ein binäres Bild berechnet, siehe Abb.3a. Aus dem Binärbild wird am Tiefpunkt der Schleifscheibe die Dicke der anliegenden KSSStrömung bestimmt, da im Schleifprozess an dieser Position der Schleifspalt zwischen Schleifscheibe und Werkstück liegen würde und somit an dieser Position der KSS in den Schleifprozess eingebracht wird. Bei der verwendeten Methode zur Messung der Schichtdicke kann aufgrund der nahe an der KSS-Schicht liegenden Tropfen eine etwas breiter gemessene Schichtdicke resultieren. Somit ist die gemessene KSS-Schichtdicke von $640 \mu \mathrm{m}$ als großzügige Abschätzung zu interpretieren. Um die Strömungsgeschwindigkeit der KSS-Schicht zu messen, ist entsprechend eine räumliche Auflösung von kleiner $640 \mu \mathrm{m}$ erforderlich. Bei den verwendeten größeren Auswertefenstern des Kreuzkorrelationsauswertealgorithmus ist demnach ein Mittelungseffekt mit über und unter der KSSSchicht liegenden Bereichen zu erwarten.

Für die Detektion der Schleifscheibenoberfläche werden die im Binärbild in negativer $y$-Richtung als erstes auftretenden Gradienten mittels Gaußfilter mit einer Standardabweichung von $0,8 \mathrm{~mm}$ verbreitert, siehe Abb. 3b. An die so ermittelte Kontur wird die Schleifscheibenoberfläche über den Kreisumfang mit dem Schleifscheiben- 

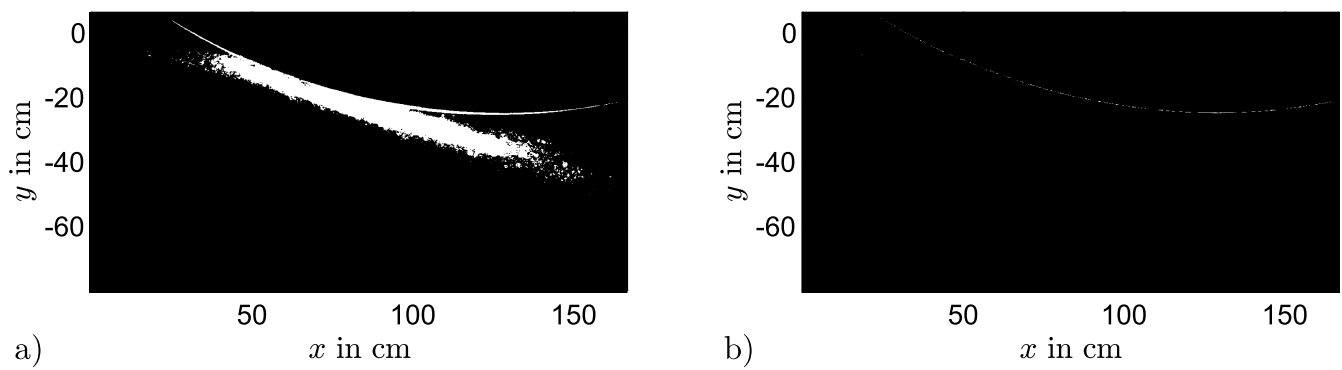

Abb. 3: a) Binarisierung von 60 summierten PIV-Bildern mit dem Schwellenwert nach Otsu [13]. b) Gaußgefilterte Kontur der ermittelten Schleifscheibenoberfläche.

radius von $20 \mathrm{~cm}$ approximiert. Der so bestimmte Mittelpunkt der Schleifscheibe liegt bei $x_{\mathrm{SS}}=110,7 \mathrm{~cm}, y_{\mathrm{SS}}=$ $175,6 \mathrm{~cm}$. Um die im PIV-Koordinatensystem ermittelte Schleifscheibenoberfläche in das SIV-Koordinatensystem $\mathrm{zu}$ übertragen, wird im Bereich (PIV-Koordinaten) von $x=[70 \mathrm{~cm}, 140 \mathrm{~cm}], y=[-30 \mathrm{~cm},-40 \mathrm{~cm}]$ die Verschiebung der SIV-Messkoordinaten gesucht, für die die SIVStrömungsfelder optimal den PIV-Strömungsfeldern gleichen. Als Optimierungsparameter wird dafür das Differenzquadrat der Geschwindigkeitsfelder zwischen PIVund SIV-Messung minimiert. Der angegebene Koordinatenbereich wird gewählt, da hier vergleichsweise niedrige Messunsicherheiten in beiden Datensätzen auftreten. Die so übereinander gelegten Messkoordinaten ermöglichen einen quantitativen Vergleich der PIV- und SIVMessungen.

Für die Bestimmung der Strömungsfelder werden 700 PIV- und 1000 SIV-Einzelmessungen gemittelt. Ein kommerzieller PIV-Auswertealgorithmus mit adaptiver Auswertefenstergröße mit minimaler Größe von $32 \mathrm{px} \times 32 \mathrm{px}$ in einem Raster von $16 \mathrm{px} \times 16 \mathrm{px}$ berechnet die Geschwindigkeitsfelder. Aus dem Raster ergibt sich eine räumliche Auflösung für die PIV-Messungen von 1,28 mm und für die SIV-Messungen von 2,40 mm, was für eine Messung der Strömungsgeschwindigkeit der an der Schleifscheibe anliegenden KSS-Schicht mit einer Dicke von $640 \mu \mathrm{m}$ nicht ausreicht. Aufgrund der niedrigeren Ortsauflösung tritt somit ein Mittelungseffekt des anliegenden Strömungsfilms auf. Abbildung 4 zeigt die gemessenen Strömungsfelder $\vec{v}_{\text {PIV }}$ mit PIV und $\vec{v}_{\text {SIV }}$ mit SIV für die untersuchten Volumenströme $Q$. Im Rahmen der zweifachen Standardunsicherheit der gemessenen Geschwindigkeitswerte erzielen die PIV- und SIV-Messungen im Wesentlichen die gleichen Ergebnisse. Abbildung 5 zeigt weiß markiert die Bereiche im Strömungsfeld, bei denen die gemessenen Geschwindigkeitswerte der PIV- und SIV-Messungen im Rahmen der zweifachen Standardabweichung nicht übereinstimmen. Im übereinstimmenden Bereich ergeben sich im
Mittel Standardabweichungen von $0,1 \mathrm{~m} \mathrm{~s}^{-1}$ bis $0,3 \mathrm{~m} \mathrm{~s}^{-1}$ für PIV und von $0,4 \mathrm{~m} \mathrm{~s}^{-1}$ bis $0,8 \mathrm{~m} \mathrm{~s}^{-1}$ für SIV abhängig vom Volumenstrom, wobei bei steigendem Volumenstrom die Standardabweichung zunimmt. Es zeigt sich, dass besonders für höhere Volumenströme im Bereich von $x \approx[30 \mathrm{~cm}, 70 \mathrm{~cm}], y \approx[-10 \mathrm{~cm},-20 \mathrm{~cm}]$ quantitative Unterschiede bestehen. Grund dafür ist, dass der Kontrast in den SIV-Bildern in diesem Bereich nicht für eine Messung der Verschiebung der Strömungsstrukturen ausreicht, da für höhere Volumenströme die KSS-Strömung hier eine zu hohe Tiefe besitzt, so dass nicht genug Lichtintensität vom Hintergrund in Richtung Kamera gelangt, vgl. Abb. 1c. Im schleifscheibennahen Bereich sind mit einer Lichtschnittbeleuchtung somit präzisere Messergebnisse zu erlangen als mithilfe von Schattenwurfbildern. Jedoch bleibt zu klären, inwieweit der Lichtschnitt aufgrund von Lichtbrechungen an dem Phasenübergang zwischen Luft und KSS aufgeweitet wird. In den Geschwindigkeitsfeldern in Abb. 4 sind zusätzlich acht Isotachen eingezeichnet, welche sich aus dem Betrag der Vektordifferenz $\left|\vec{v}_{\text {PIV,SIV }}-\vec{v}_{t}\right|$ zwischen den gemessenen Geschwindigkeitsfeldern $\vec{v}_{\text {PIV,SIV }}$ und der tangentialen Schleifscheibenumfangsgeschwindigkeit $\left|\vec{v}_{\mathrm{t}}\right|=25 \mathrm{~m} \mathrm{~s}^{-1}$ berechnen. Die Richtung und der Betrag der tangentialen Schleifscheibenumfangsgeschwindigkeit bleibt dabei in radialer Richtung zum Schleifscheibenmittelpunkt konstant. Für die unterschiedlichen Volumenströme sind die acht Isotachen im jeweiligen Wertebereich der Geschwindigkeitsdifferenzen äquidistant verteilt. Abhängig von dem eingestellten Volumenstrom verdeutlichen die Isotachen die KSS-Mitnahme an der Schleifscheibe. Bei parallelem Verlauf der Isotachen zur Schleifscheibenoberfläche scheint der KSS der Schleifscheibe homogen zu folgen, was besonders für einen Volumenstrom von $31,9 \mathrm{~L} \mathrm{~min}^{-1}$ näherungsweise in den PIV-Messergebnissen zu erkennen ist. Für höhere Volumenströme ist ein Abprallen des KSS-Strahls durch einen gebeugten Verlauf in reflektierender Richtung der KSS-Anströmung an der Schleifscheibe zu ver- 

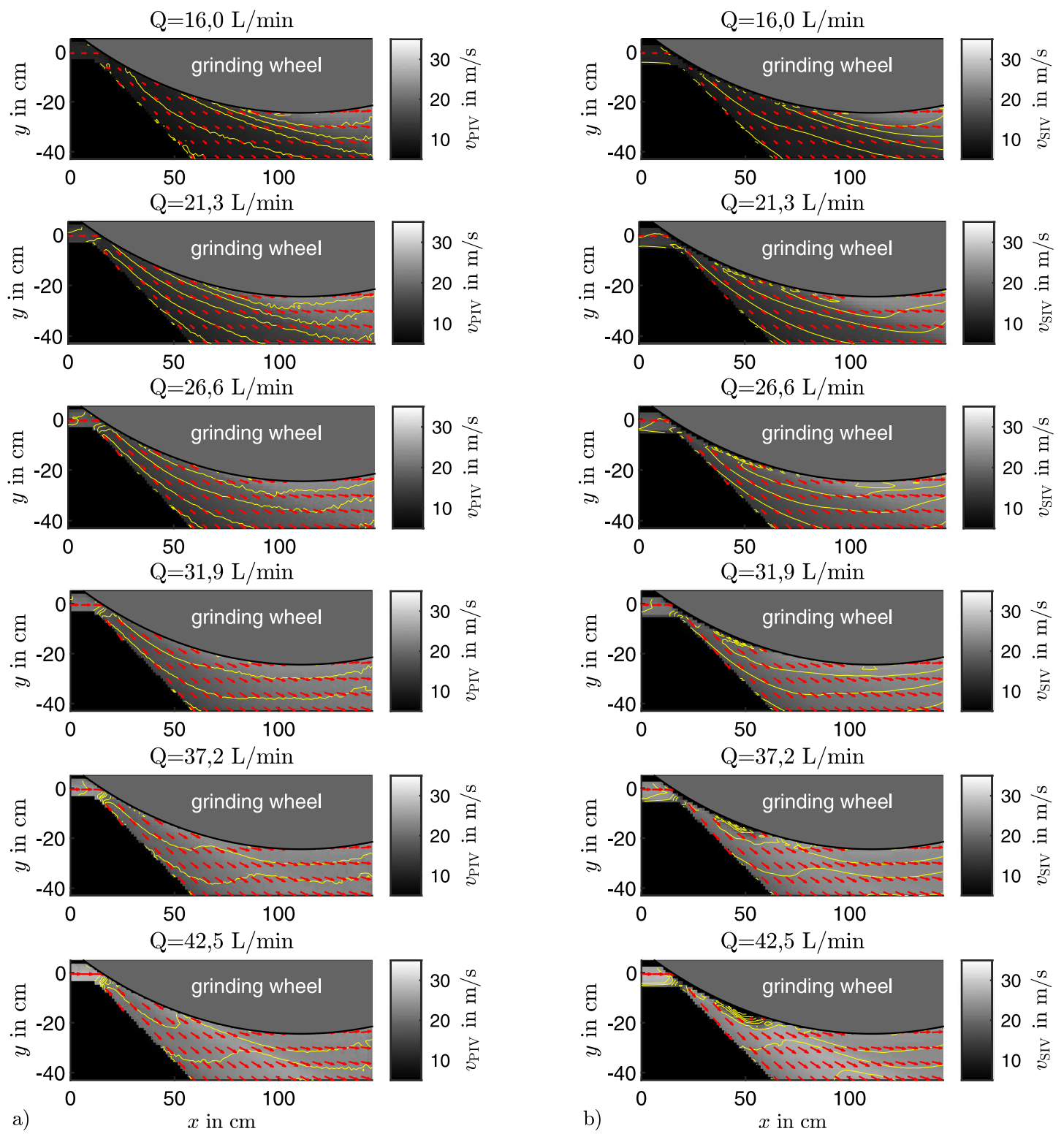

Abb. 4: Mittels a) PIV und b) SIV gemessene zeitgemittelte Strömungsfelder der KSS-Zufuhr an der Schleifscheibe bei verschiedenen KSSVolumenströmen $Q=(16,0 ; 21,3 ; 26,6 ; 31,9 ; 37,2 ; 42,5) \mathrm{L} \mathrm{min}^{-1}$. Die Beträge der gemessenen Strömungsfelder $v_{\mathrm{PIV}}$ und $v_{\mathrm{SIV}} \operatorname{sind}$ in Graustufen visualisiert. Zusätzlich sind in gelb acht äquidistant verteilte Isotachen eingezeichnet.

muten. Für niedrigere Volumenströme ist eine Beschleunigung des KSS an der Schleifscheibe zu erkennen, da die Isotachen gestaffelt nach erhöhter KSS-Lauflänge direkt an der Schleifscheibe nahezu tangential zur Schleifscheibenoberfläche verlaufen. Es lässt sich somit vermuten, dass eine optimale KSS-Mitnahme an der Schleifscheibe bei einem Volumenstrom von etwa 31,9 $\mathrm{L} \mathrm{min}^{-1}$ erreicht sein könnte.

In der Nähe der Schleifscheibe muss für die Geschwindigkeitsmessungen durch ein tieferes KSS-Volumen zwischen Beleuchtungslichtschnitt bzw. Hintergrundbe- leuchtung und Kamera geschaut werden, was optische Störungen verursacht und damit die Bestimmung eines eindeutiges Korrelationsmaximums erschwert. Um zu klären, inwieweit schleifscheibennahe Strömungsgeschwindigkeitsmessungen mit dem gewählten Messaufbau möglich sind, werden die minimalen Abstände zwischen der Position der gemessenen Geschwindigkeitswerte und der Schleifscheibenoberfläche bestimmt. Dabei werden lediglich Geschwindigkeitswerte berücksichtigt, die nicht kleiner als $1 \mathrm{~m} \mathrm{~s}^{-1}$ unterhalb der Anströmgeschwindigkeit bei $(x, y) \approx(0,0)$ oder, sollte die Anströmgeschwindigkeit 

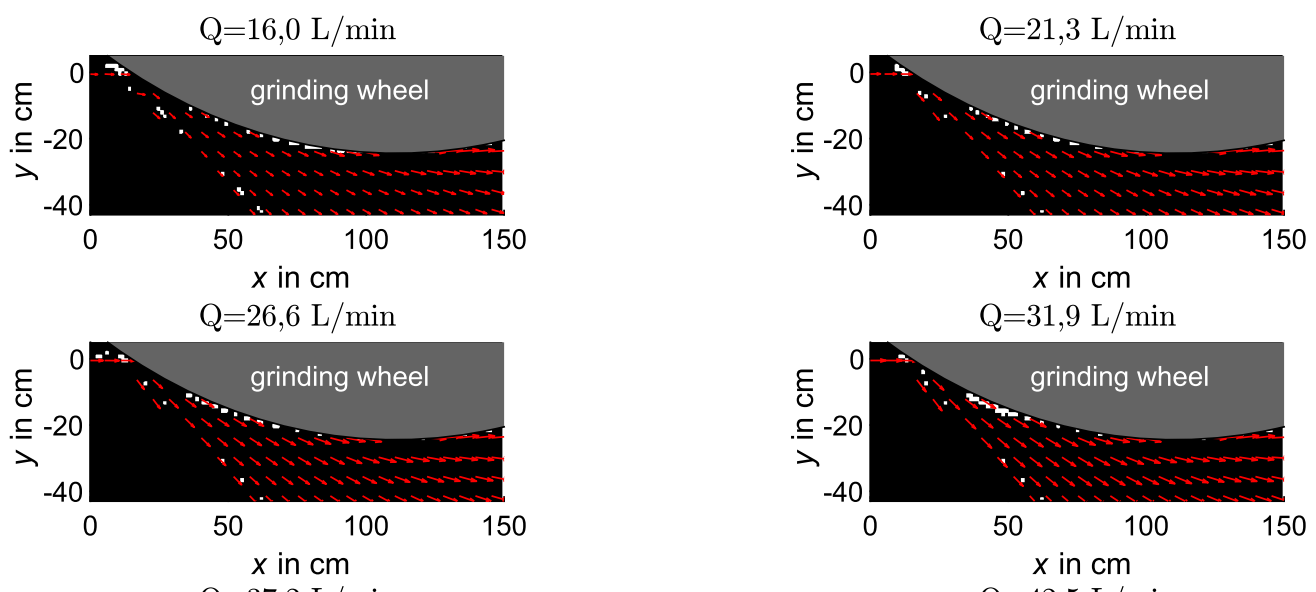

$\mathrm{Q}=37,2 \mathrm{~L} / \mathrm{min}$
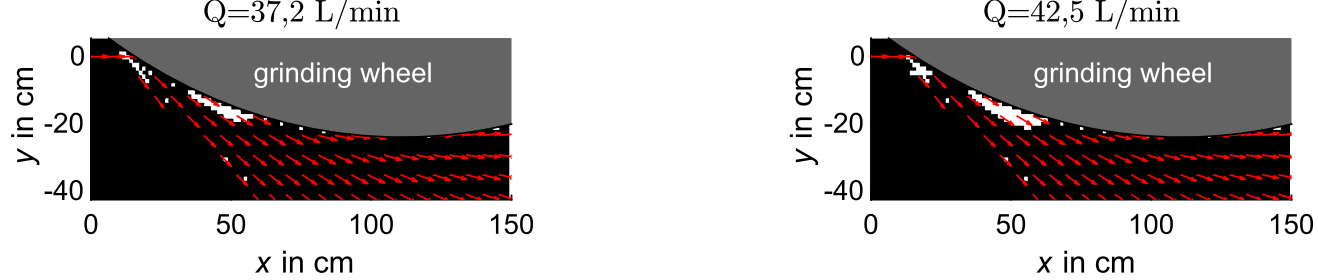

Abb. 5: Binäre Darstellung der Absoluten Differenz zwischen den PIV- und SIV-Messdaten für verschiedene KSS-Volumenströme $Q=$ $(16,0 ; 21,3 ; 26,6 ; 31,9 ; 37,2 ; 42,5) \mathrm{L} \mathrm{min}^{-1}$. An den weiß markierten Stellen stimmen die Messergebnisse im Rahmen der zweifachen Standardunsicherheit nicht überein. Die roten Pfeile deuten das mittels PIV gemessene Strömungsfeld an, wobei die Skalierung der von Abb. 4 entspricht.

höher liegen als die Schleifscheibenumfangsgeschwindigkeit, kleiner als $1 \mathrm{~m} \mathrm{~s}^{-1}$ unterhalb der Schleifscheibenumfangsgeschwindigkeit sind. Diese Grenze wird aufgrund der Tatsache gesetzt, dass schleifscheibennah nicht wesentlich niedrigere Geschwindigkeitswerte erwartet werden können. Eine Ausnahme dazu stellt der Bereich des Staupunktes im Auftreffbereich der KSS-Strömung auf die Schleifscheibe dar. Daher wird dieser Bereich bei dieser Betrachtung nicht berücksichtigt. Es ist jedoch zu erwarten, dass dieser Bereich eine untergeordnete Rolle für die KSS-Zufuhr für den Schleifprozess spielt, weil hier kaum ein Beitrag zum KSS-Transport in Richtung des späteren Schleifspalts erbracht wird. Abbildung 6 zeigt den ermittelten minimalen Abstand $r(x)$ für die PIV- und SIVMessungen für die untersuchten Volumenströme $Q$. Für die minimalen radialen Abstände $r$ werden die schleifscheibennähesten Geschwindigkeitswerte verwendet. Der sägezahnähnliche Verlauf von $r$ über der Position $x$ resultiert aus der Rasteranordnung von $16 \mathrm{px} \times 16 \mathrm{px}$ der Auswertefenster des Auswertealgorithmus für die Geschwindigkeitsauswertung. Die negativen Abstände resultieren daraus, dass für die Geschwindigkeitsauswertung der Schwerpunkt der Auswertefenster mit einer minimalen Größe von 2,5 $\mathrm{mm} \times 2,5 \mathrm{~mm}$ bei PIV und 4,8 $\mathrm{mm} \times 4,8 \mathrm{~mm}$ bei SIV teilweise innerhalb der Schleifscheibe liegen. Im
Bereich von $x<70 \mathrm{~cm}$ ist zu erkennen, dass mittels PIV gleich nahe oder zumeist nähere Messwerte zur Schleifscheibenoberfläche als mittels SIV erreicht werden. Die Diskrepanz zwischen PIV und SIV steigt in diesem Bereich für höhere Volumenströme an, was auf den Kontrastverlust in den Schattenwurfbildern aufgrund der zunehmenden KSS-Strömungstiefe zurückzuführen ist. Letztlich werden mittels PIV Geschwindigkeiten mit minimalen Abständen zwischen $0 \mathrm{~cm}$ und $2 \mathrm{~cm}$ und mittels SIV zwischen $0 \mathrm{~cm}$ und $5 \mathrm{~cm}$ gemessen. Inwieweit die Auflösung der Strömungsgeschwindigkeitsverteilung des anhaftenden KSS-Film an der Schleifscheibe möglich ist, muss für Messungen mit erhöhter optischer Auflösung geprüft werden. Die Ergebnisse zeigen jedoch, dass zumindest räumlich gemittelte Messungen der schleifscheibenanhaftenden KSS-Strömung möglich sind.

Aus den gemessenen Geschwindigkeiten im Anströmbereich bei $(x, y) \approx(0,0)$ kann erstmals direkt das Geschwindigkeitsverhältnis $v=v_{\mathrm{KSS}} / v_{\mathrm{SS}}$ zwischen Anströmungsgeschwindigkeit $v_{\mathrm{KSS}}$ und Schleifscheibenumfangsgeschwindigkeit $v_{\mathrm{SS}}$ gemessen werden, wofür bislang lediglich theoretisch abgeschätzte Werte aus dem eingestellten Volumenstrom und der Düsenaustrittsfläche genutzt wurden. Tabelle 1 listet die gemessenen Geschwindigkeitsverhältnisse $v$ für die untersuchten Volumenströme $Q$. Ge- 

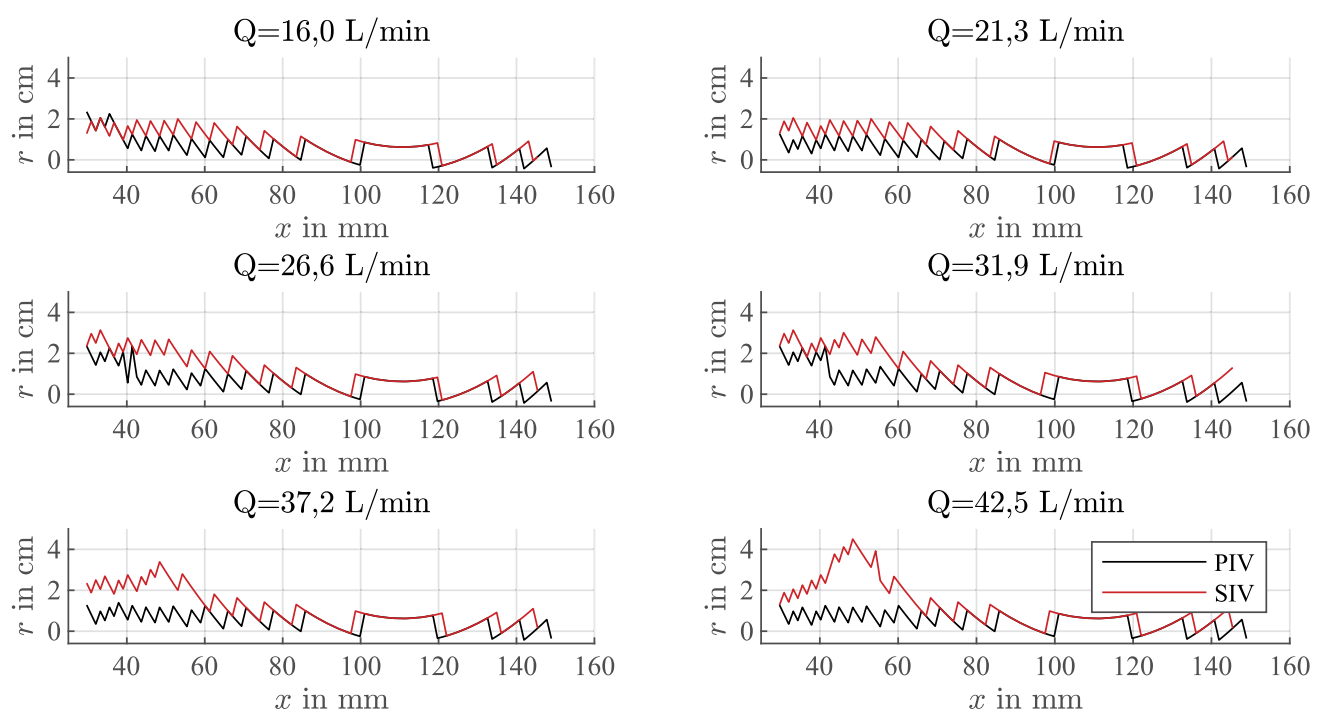

Abb. 6: Minimaler radialer Abstand $r$ zwischen den gemessenen Geschwindigkeitsfeldern mittels PIV und SIV und der Schleifscheibenoberfläche für die KSS-Volumenströme $Q=(16,0 ; 21,3 ; 26,6 ; 31,9 ; 37,2 ; 42,5) \mathrm{L} \mathrm{min}^{-1}$.

Tab. 1: Die gemessenen Geschwindigkeitsverhältnisse $v$ zwischen KSS-Anströmgeschwindigkeit und Schleifscheibenoberflächengeschwindigkeit für die untersuchten Volumenströme $Q$.

\begin{tabular}{lrrrrrr}
\hline$Q$ in $\mathrm{min}^{-1}$ & $\mathbf{1 6 , 6}$ & $\mathbf{2 1 , 3}$ & $\mathbf{2 6 , 6}$ & $\mathbf{3 1 , 9}$ & $\mathbf{3 7 , 2}$ & $\mathbf{4 2 , 5}$ \\
\hline$v$ & $0,438 \pm 0,001$ & $0,584 \pm 0,001$ & $0,715 \pm 0,001$ & $0,839 \pm 0,003$ & $0,977 \pm 0,001$ & $1,107 \pm 0,001$ \\
\hline
\end{tabular}

mäß einer heuristischen Optimierungsstudie für die KSSZufuhr in Abhängigkeit des Geschwindigkeitsverhältnisses zwischen KSS und Schleifscheibe ist ein Verhältnis von etwa 0,8 zu präferieren [1], was hier zwischen einem Volumenstrom von $Q=26,6 \mathrm{~L} \mathrm{~min}^{-1}$ und $Q=31,9 \mathrm{~L} \mathrm{~min}^{-1}$ erreicht wird. Da für höhere Geschwindigkeitsverhältnisse ein Abprallen des KSS-Strahls anhand der Isolinien in Abb. 4 sichtbar wird, wird die Hypothese aufgestellt, dass dieses Abprallen des Kühlschmierstoffes für eine Verringerung der Kühleffizienz verantwortlich ist.

\section{Zusammenfassung und Ausblick}

Schleifen ist ein wichtiger Fertigungsprozess, bei dem Kühlschmierstoff (KSS) eingesetzt wird, um Schädigungen am Werkstück durch Schleifbrand zu verhindern. Eine Optimierung der KSS-Zufuhr würde die Oberflächengüte verbessern und eine Rohstoffschonung bewirken. Bislang wurden jedoch nur heuristische Strömungsoptimierungen durchgeführt. Um eine zielgerichtete Optimierung der KSS-Strömung zu ermöglichen, werden die optischen Strömungsfeldmessverfahren Particle Image Velocimetry
(PIV) und Schattenwurf Image Velocimetry (SIV) in einem ersten Schritt prozessnah, d.h. in einer kommerziellen Schleifmaschine bei rotierender Schleifscheibe, zunächst aber ohne Werkstück zum Einsatz gebracht.

Die KSS-Zufuhr wird in der Schleifmaschine über eine kühleffiziente Rouse-Düse realisiert [1]. Es werden PIV- und SIV-Messungen der KSS-Strömung in der Umgebung der rotierenden Schleifscheibe durchgeführt. Abgesehen vom Auftreffbereich der KSS-Anströmung nahe der Schleifscheibe konnten mittels PIV und SIV unter Verwendung der Strömungsstrukturen als Tracer die KSSStrömungsfelder erfolgreich gemessen werden. Es zeigt sich, dass die Schattenwurfbilder im Auftreffbereich nahe der Schleifscheibe zu wenig Kontrast für die Geschwindigkeitsauswertung liefern, was besonders für Volumenströme ab $37 \mathrm{~L} \mathrm{~min}^{-1} \mathrm{zu}$ einer Abnahme des Signal zu Rauschverhältnis und damit zu einem Anstieg der Messunsicherheit führt. Die Position der Schleifscheibe konnte mittels Bildauswertung aus den PIV-Bildern extrahiert und somit die Schichtdicke der anhaftenden Strömung an der Schleifscheibe mit $640 \mu \mathrm{m}$ bestimmt werden. Mit der räumlichen Auflösung von 1,28 mm des PIV- bzw. 2,40 mm des SIV-Messsystems wird mit dem hier gewählten Messaufbau die Geschwindigkeit der anhaftenden 
Strömung nicht örtlich aufgelöst, sondern in diesem Bereich ein mittlerer Strömungsgeschwindigkeitswert ermittelt. Ob die Geschwindigkeitsverteilung der anhaftenden KSS-Strömung einen signifikanten Einfluss auf die Kühleffizienz hat, lässt sich dadurch mit dem gewählten Messaufbau nicht abschließend klären. Es lässt sich jedoch vermuten, dass besonders das Volumen der anhaftenden KSSStrömung einen dominanten Einfluss auf die Kühleffizienz hat. Denn es ist davon auszugehen, dass besonders ein erhöhtes anhaftendes KSS-Volumen zu einem erhöhten Transport von KSS in den Schleifprozess im Schleifspalt zwischen Schleifscheibe und Werkstück und damit zu einer erhöhten Kühleffizienz sorgt. Der minimale Abstand der Geschwindigkeitsfeldmessungen zur Schleifscheibenoberfläche beträgt $0 \mathrm{~cm}$ bis $2 \mathrm{~cm}$ für PIV und $0 \mathrm{~cm}$ bis $5 \mathrm{~cm}$ für SIV, wobei die minimal erreichbaren Abstände für SIV mit dem verwendeten KSS-Volumenstrom im Auftreffbereich auf die Schleifscheibe steigen. Somit ist besonders die bei den PIV-Messungen genutzte Laserlichtschnittbeleuchtung für die Geschwindigkeitsfeldmessung der KSSZufuhr beim Schleifen geeignet.

Mit den präsentierten Verfahren kann nun auch das Geschwindigkeitsverhältnis zwischen KSS-Anströmung und Schleifscheibenoberfläche experimentell gemessen werden. Im Ergebnis wird eine Verringerung der Kühleffizienz für Geschwindigkeitsverhältnisse ab 0,977 [1] auf ein Abprallen des KSS-Strahls an der Schleifscheibenoberfläche zurückgeführt.

Ausblickend sind Messungen an einem Analogieversuchsstand geplant, bei dem prozessäquivalent die Kühlleistung der KSS-Strömung gemessen werden kann. Durch die Einbringung eines Werkstücks verschlechtert sich jedoch die optische Zugänglichkeit aufgrund einer deutlichen Zunahme an KSS-Tropfen im optischen Beobachtungs- und Beleuchtungspfad. Deshalb ist zu klären, ob und mit welcher Güte unter diesen erschwerten Randbedingungen weiterhin optische Strömungsfeldmessungen durchgeführt werden können.

Finanzierung: Gefördert durch die Deutsche Forschungsgemeinschaft (DFG) - 415003387.

\section{Literatur}

1. C. Heinzel, D. Meyer, B. Kolkwitz, and J. Eckebrecht. Advanced approach for a demand-oriented fluid supply in grinding. CIRP Annals, 64(1):333-336, 2015.

2. E. Brinksmeier, D. Meyer, A. Huesmann-Cordes, and C. Herrmann. Metalworking fluids-mechanisms and performance. CIRP Annals, 64(2):605-628, 2015.
3. C. Heinzel, B. Kirsch, D. Meyer, and J. Webster, Interactions of grinding tool and supplied fluid. CIRP Annals, 64(2):624-645, 2020.

4. A. Fischer. imaging flow velocimetry with laser Mie scattering. Applied Sciences, 7(12):1298, 2017.

5. A. Fischer. Model-based review of Doppler global velocimetry techniques with laser frequency modulation. Optics and Lasers in Engineering, 93:19-35, 2017.

6. M. Raffel, C. Willert, and J. Kompenhans. Particle Image Velocimetry: A Practical Guide (Experimental Fluid Mechanics). Springer, 2002.

7. Z.-M. Cao, K. Nishino, S. Mizuno, and K. Torii. PIV measurement of internal structure of diesel fuel spray. Experiments in Fluids, 29(7):211-219, 2000.

8. T. D. Nguyen, J. C. Wells, and C. V. Nguyen. Velocity measurement of near-wall flow over inclined and curved boundaries by extended interfacial particle image velocimetry. Flow Measurement and Instrumentation, 23(1):33-39, 2012.

9. D. T. Squire, C. Morrill-Winter, N. Hutchins, I. Marusic, M. P. Schultz, and J. C. Klewicki. Smooth- and rough-wall boundary layer structure from high spatial range particle image velocimetry. Physical Review Fluids, 1(6):064402, 2016.

10. L. C. Jia, Y. D. Zhu, Y. X. Jia, H. J. Yuan, and C. B. Lee. Image pre-processing method for near-wall PIV measurements over moving curved interfaces. Measurement Science and Technology, 28(3):035201, 2017.

11. D. R. Jonassen, G. S. Settles, and M. D. Tronosky. Schlieren "PIV" for turbulent flows. Optics and Lasers in Engineering, 44(3-4):190-207, 2006.

12. P. Geilert, C. Heinzel, and A. Wagner. Grinding fluid jet characteristics and their effect on a gear profile grinding process. Inventions, 2(4):27, 2017.

13. N. Otsu. A threshold selection method from gray-level histograms. IEEE Transactions on Systems, Man, and Cybernetics, 9(1):62-66, 1979.

\section{Autoreninformationen}

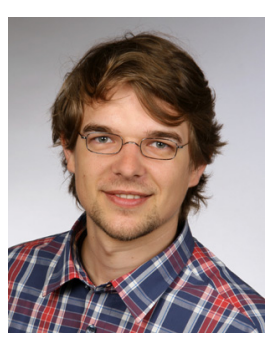

\section{Christoph Vanselow}

Universität Bremen, Bremer Institut für Messtechnik, Automatisierung und Qualitätswissenschaft (BIMAQ), Linzer Str. 13, 28359 Bremen, Germany c.vanselow@bimaq.de

Christoph Vanselow ist seit 2016 wissenschaftlicher Mitarbeiter an der Universität Bremen am Bremer Institut für Messtechnik, Automatisierung und Qualitätswissenschaft (BIMAQ) im Fachbereich Produktionstechnik. Zuvor absolvierte er sein Physikstudium ebenfalls an der Universität Bremen. Seit seiner Anstellung am BIMAQ forscht er im Bereich der optischen Strömungsmesstechnik. 


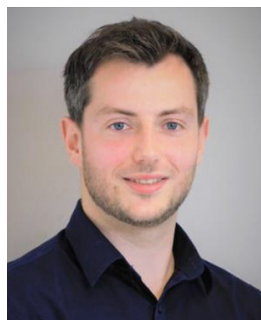

\section{Björn Espenhahn}

Universität Bremen, Bremer Institut für

Messtechnik, Automatisierung und Qualitätswissenschaft (BIMAQ), Linzer Str. 13, 28359 Bremen, Germany

b.espenhahn@bimaq.de

Björn Espenhahn absolvierte ein Physik-Studium an der Universität Oldenburg 2019 und arbeitet seitdem als wissenschaftlicher Mitarbeiter an der Universität Bremen am Bremer Institut für Messtechnik, Automatisierung und Qualitätswissenschaft (BIMAQ). Dort forscht er im Bereich der optischen Strömungsmesstechnik, insbesondere an der Messbarkeit von optischen Messtechniken an Flüssigkeiten mit fluktuierenden Oberflächen.

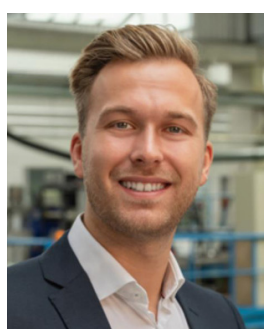

\section{Lukas Schumski}

Leibniz-Institut für Werkstofforientierte Technologien (IWT), Badgasteiner Straße 3, 28359 Bremen, Germany schumski@iwt-bremen.de

Lukas Schumski studierte von 2012 bis 2019 an der Universität Bremen im Fachbereich Produktionstechnik. Während der Zeit war er bereits als studentische Hilfskraft von 2013-2019 am LeibnizInstitut für werkstofforientierte Technologien (IWT) - Bremen im Bereich Fertigungstechnik tätig. Seit 2019 mit dem Masterabschluss setzte er seine wissenschaftliche Karriere im Leibniz-Institut für werkstofforientierte Technologien - Bremen als wissenschaftlicher Mitarbeiter mit dem Ziel einer Promotion fort.

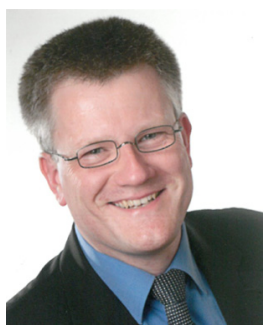

\section{Dirk Stöbener}

Universität Bremen, Bremer Institut für Messtechnik, Automatisierung und Qualitätswissenschaft (BIMAQ), Linzer Str. 13, 28359 Bremen, Germany Universität Bremen, MAPEX Center for Materials and Processe, Postbox 330440 , 28359 Bremen, Germany d.stoebener@bimaq.de

Dirk Stöbener absolvierte ein Physik-Studium an der Universität Bremen und ist dort auch im Fachbereich Produktionstechnik als Leiter der Arbeitsgruppe „In-prozess Messtechnik und Optik“ am Bremer Institut für Messtechnik, Automatisierung und Qualitätswissenschaft (BIMAQ) tätig. Seine Forschungsinteressen umfassen fertigungsnahe Messverfahren z. B. zur Positionsbestimmung und zur Charakterisierung von (geometrischen) Werkstückeigenschaften.

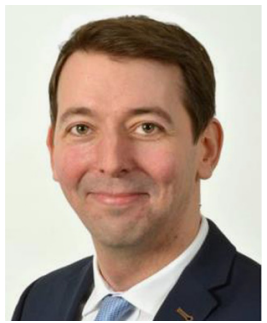

\section{Daniel Meyer}

Leibniz-Institut für Werkstofforientierte Technologien (IWT), Badgasteiner Straße 3, 28359 Bremen, Germany Universität Bremen, MAPEX Center for Materials and Processe, Postbox 330440 , 28359 Bremen, Germany

dmeyer@iwt.uni-bremen.de

Daniel Meyer ist seit 2006 wissenschaftlicher Mitarbeiter am IWT Bremen und promovierte im Jahr 2012 im Bereich Fertigungstechnik. Seine Dissertationsschrift behandelt die fertigungstechnischen Möglichkeiten zur mechanisch induzierten Gefügeumwandlung in metastabilen Restausteniten. Seit 2012 ist er als Oberingenieur sowie als Leiter einer internen Nachwuchsforschungsgruppe in der Projektverantwortung sowie in der Hochschullehre tätig. 2016/2017 war er als Gastwissenschaftler und -dozent an der University of North Carolina at Charlotte (UNCC), USA.

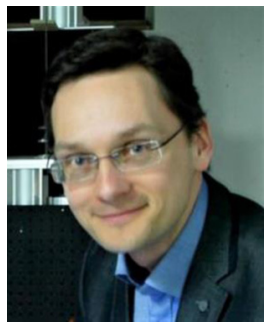

\author{
Andreas Fischer \\ Universität Bremen, Bremer Institut für \\ Messtechnik, Automatisierung und \\ Qualitätswissenschaft (BIMAQ), Linzer Str. \\ 13, 28359 Bremen, Germany \\ Universität Bremen, MAPEX Center for \\ Materials and Processe, Postbox 330440, \\ 28359 Bremen, Germany \\ andreas.fischer@bimaq.de
}

Prof. Dr.-Ing. habil. Andreas Fischer übernahm 2016 die Leitung des Bremer Instituts für Messtechnik, Automatisierung und Qualitätswissenschaft (BIMAQ) am Fachbereich Produktionstechnik der Universität Bremen. Nach dem Studium der Elektrotechnik und der daran anschließenden Promotion, für die er u. a. 2010 mit dem Messtechnik-Preis der AHMT e. V. ausgezeichnet wurde, habilitierte er sich auf dem Gebiet der Messtechnik am Institut für Mess- und Sensorsystemtechnik der Fakultät Elektrotechnik und Informationstechnik an der Technischen Universität Dresden. Zu seinen Forschungsinteressen zählen laseroptische Messsysteme für hochdynamische Strömungs- und Fertigungsprozesse sowie messsystemtheoretische Ansätze zur Beschreibung von Messbarkeitsgrenzen. 